\title{
The Friction and Wear Influence of Laser Processing Bionic Brake Drum Unit on the Brake Pad
}

\author{
Haiyan $\mathrm{Hu}^{1}$, Haiyang Yang ${ }^{2, *}$, Jiandong Yang ${ }^{3}$, Qingnian Wang ${ }^{4}$, Ti Zhou ${ }^{5}$ \\ 1,3 Jilin Business and Technology College, Department of Engineering, Changchun, China \\ ${ }^{2,4}$ Jilin University, College of Automotive Engineering, Changchun, China \\ 5 Jilin University, College of Mechanical Science and Aerospace Engineering, Changchun, China
}

\begin{abstract}
This article starts with the analysis of the contact between the laser processing bionic brake drum unit body and the brake pad and performs the analysis of the force between the unit body and the brake pad based on elastic mechanics and contact mechanics. By using the Preston equation, the wear of the brake pad caused by the unit body is obtained, as well as the relevant factors and influence rule which affects the wear of the brake pad are found. During laser processing on the bionic brake drum unit body, in order to reduce wear of brake pad, the height of the unit body should be minimized. Besides, the light intensity at the centre of the laser spot should be reduced, and the laser intensity distribution should be more uniform, which can effectively improve the environmental friendliness of the brake drum.
\end{abstract}

\section{Introduction}

The braking of the truck is realized by the frictional resistance between the brake drum and the brake pad. The Truck braking function depends on the friction resistance between the brake drum and the brake pad. The friction surface of the brake drum is an inner cylindrical surface, and the friction surface of the brake pad is a part of the outer cylindrical surface.

The brake drum is fixed on the truck wheel. It rotates with the wheel when the truck is moving. The brake pad is fixed on the body, which does not rotate when the truck is moving. During braking, the brake drum and the brake pad are pressed together under the action of external force. Both of them rub each other to generate frictional resistance and form a frictional resistance moment, which prevents the truck from moving and achieves the purpose of braking.

The bionic non-smooth surface with different microstructure can be processed by the laser technology on the surface of metal material. This bionic shape feature can significantly improve the wear and thermal fatigue resistance of metal material, as well as realize the perfect combination of laser processing technology and bionic treatment, which has been proved by the Bionic Wear-Resistant and Anti-Fatigue Research Group of Jilin University $[1,2]$ through an amount of experimental researches.

After the laser bionic treatment of the brake drum, many hard convex unit bodies are formed on the surface of the brake drum. Those unit bodies play an important role in the braking process which can effectively reduce the wear of the bionic brake drum and improve the brake drum service life. However, the strengthened bionic brake drum increases the wear of the brake pad, which reduces the service life of the brake pad, and increases the replacement frequency of the brake pad.

Therefore, it is necessary to study the unit body considering the friction and wear of the brake pad. While reducing the wear of the brake drum, the wear condition of the brake pad should also be taken into account to ensure the service life of the brake pad, thereby reducing the cost of truck maintenance.

Combining the actual needs of truck brake drum and considering the convenience, feasibility and manufacturing cost of processing the bionic unit body on the brake drum, the bionic unit body pattern feature observed from the surface has been set for the theoretical analysis in this study.

\section{The contact stress between the unit body and the brake pad}

Considering the large radius of the cylindrical surface where the brake drum and the brake pad rub against to each other, the friction surface is considered as a plane. Correspondingly, in order to facilitate the analysis, the friction is supposed as the plane friction.

Based on this condition, in the braking process, the unit body on the brake drum and the friction surface of the brake pad are pressed against each other under the action of the external force. The elastic deformation and non-planar contact will be generated between two friction surfaces consequently.

Therefore, the friction between the brake drum and the brake pad is no longer pure plane friction, and its friction mechanism is completely different from the traditional plane friction. Since the unit body on the

\footnotetext{
* Corresponding author: hyyang14@mails.jlu.edu.cn
} 
brake drum is generated by laser processing, considering that the laser processing spot intensity is based on the optical axis as the symmetry axis as Gaussian distribution, we define the unit body as a Gaussian revolution surface. Besides, the surface of the brake pad is still flat. The contact between the $t$ unit body and the brake pad is the contact between the Gaussian revolution surface and the semi-infinite body. The friction between them is considered as the friction between the Gaussian revolution surface and the flat plane. The contact pressure between surfaces is no longer a simple uniformly distributed pressure of two planes. Due to the complex stress distribution status of such contact friction, the wear of the brake pad based on the unit body will be changed, which is necessary to be analysed in detail.

According to the laser principle, the laser spot intensity distribution I(r) has been defined, which is the intensity of the laser spot from the optical axis $r$ (considering the symmetry, it can also be defined as the light beam radius), as shown in below (See Figure 1):

$$
I(r)=I_{\max } e^{-\frac{2 r^{2}}{w^{2}}}
$$

Where $I(r)$ is the light intensity distribution at the distance $r$ from the optical axis in the laser spot. $I_{\max }$ is the maximum light intensity of the spot (on the axis of the laser). $w$ is the light beam radius when the light intensity is $13.5 \%$ of the maximum light intensity

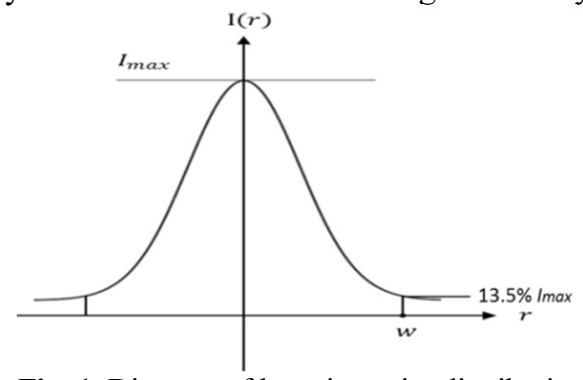

Fig. 1. Diagram of laser intensity distribution

After the bionic laser processing of the brake drum, martensite will be generated in the processed area, which forms a convex point with bigger volume. Since the intensity of the laser light irradiated at each point is different, the amount of martensite produced is different, and the height $h$ of the convex unit body is also different correspondingly as shown in Figure 2

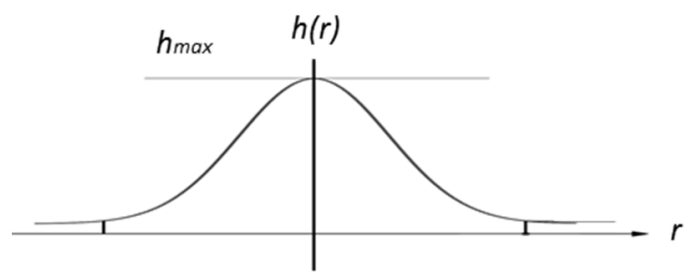

Fig. 2. Diagram of unit body

$h$ is changed with the change of laser intensity, and is a variation function of the beam radius $r$. It is assumed that the convex point height $h$ is proportional to the laser intensity received at this point, and the proportional coefficient is $C$. Considering the symmetry, the surface of the unit body is the Gaussian surface of revolution. Refer to equation (1), the $h$ is:

$$
h=C I(r)=C I_{\max } e^{-\frac{2 r^{2}}{w^{2}}}
$$

Figure 3 is a Diagram of the interaction between a unit body and the brake pad. The pressure of the unit body $P$ is perpendicular to the surface of the brake pad. $O$ is the initial contact point between the unit body and the brake pad. With the pressure $\mathrm{P}$ applied on the brake pad, the unit body and the brake pad will be deformed elastically.

Considering that the unit body is laser-treated, the unit body has a very high hardness which leads small deformation after being stressed. To facilitate the theoretical analysis, the unit body deformation is ignored. The unit body surface is still assumed as Gaussian revolution surface. By contrary, the brake pad is deformed elastically under the pressure of the unit body which forms the dent inward, and the point $O$ will be moved downward accordingly, as shown in Figure 4. The plane surface of the brake pad is deformed under the pressure of the unit body into a concave Gaussian revolution surface, which is same as the surface of the unit body.

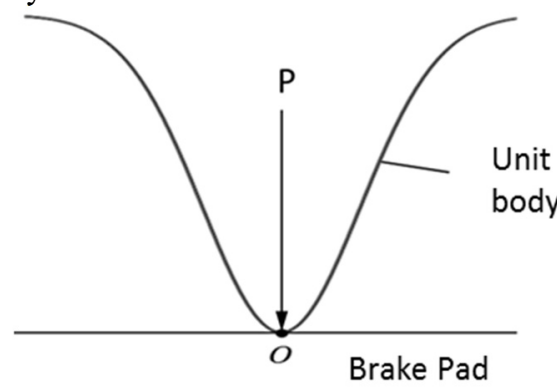

Fig. 3. The interaction between the unit body and the brake pad

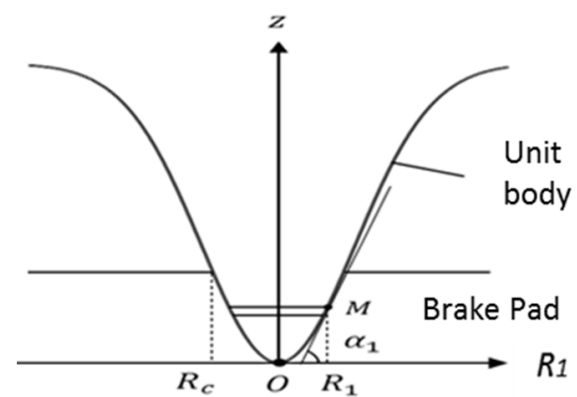

Fig. 4. Diagram of brake pad deformation under the unit body pressure

$R_{C}$ is the radius of the contact surface between the unit body and the brake pad. The point $O$ is defined as the original point of the coordinate system where the R 1 is the lateral axis which indicating the distance from the $\mathrm{Z}$ axis, and $\mathrm{Z}$ is longitudinal axis which is the axis of the unit body. Based on the Figure 1, according to equation (2), the generatrix equation of the unit body profile can be obtained as below

$$
Z=C I\left(R_{1}\right)=C I_{\max }\left(1-e^{-\frac{2 R_{1}^{2}}{w^{2}}}\right)
$$

Since the deformation of each point on the contact surface of the brake pad and the unit body is different, the contact stress on the contact surface is different as 
well. $F$ is defined as the contact stress between the unit body and the contact surface of the brake pad. Considering the symmetry, the contact stress $F$ at any point $M$ on the contact surface is only related to $R_{I}$ which is the distance from the $Z$ axis.

$Q_{0}$ is the contact stress at the lowest position which is point $O$ on the contact surface between the unit body and the brake pad.

Considering that the $O$ point on the brake pad has the biggest displacement and deformation during braking, the contact stress $Q_{0}$ of the $O$ point is also the maximum contact stress between the two contact surfaces.

According to elastic mechanics [3], the contact stress of a point $M$ on the spherical surface of the unit body and the brake pad is

$$
F=\frac{Q_{0}}{R_{c}} \sqrt{R_{c}^{2}-R_{1}^{2}}
$$

\section{Friction and wear of the unit body on the brake pad}

When analysing the friction and wear of the unit body on the brake pad, the Preston equation [4] can be referred to calculate the amount of abrasive removal wear. This equation is defined that the material removal rate per unit time of friction pair is proportional to the pressure and instantaneous relative velocity, as shown below:

$$
\Delta Z=\int_{0}^{t} k v p_{n} d t
$$

where:

$\Delta Z \quad$ The amount of abrasive removal wear;

$t$-Action time of the two in friction pair;

$k$-Proportional coefficient;

$v$ - Relative speed of the friction pair;

$p_{n}$ - The pressure of the interaction between the friction pair

Referring to the Figure 4 , take a point $M$ on the contact surface. The distance between point $M$ and $Z$ axis is the radius $R_{l}$. With the $M$ point, a small micro circle has been considered with the width $d R_{l}$. The value of $d R_{l}$ is extremely small. Since the point on this micro circle radius is $R_{l}$, the pressure value on this contact surface can be considered as a constant. Then the pressure $d p_{z}$ of the two surfaces on the small micro circle along the $Z$-axis direction should be the product of the contact pressure stress $F$ and its area, which is:

$$
d p_{z}=2 F \pi R_{1} d R_{1}
$$

Where the pressure $d p_{z}$ in the equation is along the $Z$ axis in the vertical direction; the pressure of the interaction between the friction pair $p_{n}$ in equation (5) is along the normal direction of the contact surface. Since the contact surface between the two pairs is not horizontal, the tangent plane of point $M$ is at an angle $\alpha_{1}$ to the horizontal plane, as shown in Figure 4.

Therefore, the direction of $p_{n}$ is not same with the direction of $d p_{z}$. The equation (6) cannot be simply substituted into equation (5) to calculate the amount of friction wear caused by the unit body on the brake pad on the micro circle. However, the vertical pressure $d p_{z}$ in the equation (6) can be used to figure out the pressure $p_{n}$ caused by the normal direction on the contact surface.
Then $p_{n}$ can be substituted into equation (5) to calculate the friction wear of the micro circle on the brake pad. Therefore, the point $M$ in Figure 4 can be partially enlarged which is shown in Figure 5.

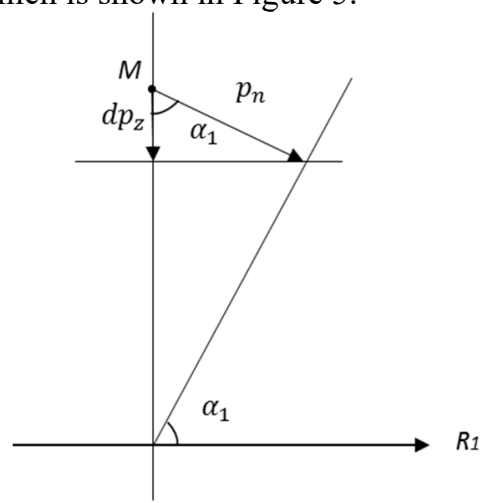

Fig. 5. Magnified diagram of force on any contact point $M$

From the Figure 5, it is shown that

$$
p_{n}=d p_{z} / \cos \alpha_{1}
$$

By substituting equation (6) into equation (7), the result is

$$
p_{n}=2 F \pi R_{1} d R_{1} / \cos \alpha_{1}
$$

By substituting equation (8) into equation (5), the result is

$$
\Delta Z_{1}=\int_{0}^{t} \frac{2 k v F \pi R_{1} d R_{1}}{\cos \alpha_{1}} d t
$$

Where, $\Delta Z_{1}$ in equation (9) is the amount of friction wear caused by the unit body on the brake pad on the micro circle. In order to calculate the wear amount $\Delta Z$ at the unit body on the brake pad at the certain time $t$, the integration is performed in equation (9) for $d R_{l}$. From the Figure 5 , it is shown that the range of $R_{I}$ is from 0 to $R_{c}$. Therefore,

$$
\Delta Z=\int_{0}^{t} \int_{0}^{R_{C}} \frac{2 k v F \pi R_{1}}{\cos \alpha_{1}} d R_{1} d t
$$

According to the trigonometric function,

$$
1 / \cos \alpha_{1}=\sqrt{1+\operatorname{tg}^{2} \alpha_{1}}
$$

Referring to the Figures 3 and 4 , considering that $\operatorname{tg} \alpha_{1}$ is the slope of the tangent on the contact surface between the unit body and the brake pad at point $M$, which is:

$$
\operatorname{tg} \alpha_{1}=\frac{d Z}{d R_{1}}
$$

By deriving from equation (3):

$$
\operatorname{tg} \alpha_{1}=\frac{d Z}{d R_{1}}=C I_{\max } \frac{4 R_{1}}{W^{2}} e^{-\frac{2 R_{1}^{2}}{W^{2}}}
$$

Then

$$
\operatorname{tg}^{2} \alpha_{1}=\frac{16 C^{2} I_{\max }^{2} R_{1}^{2}}{W^{4}} e^{-\frac{4 R_{1}^{2}}{W^{2}}}
$$

By substituting equation (13) into equation (11), the result is

$$
1 / \cos \alpha_{1}=\sqrt{1+\frac{16 C^{2} I_{\max }^{2} R_{1}^{2}}{W^{4}} e^{-\frac{4 R_{1}^{2}}{W^{2}}}}
$$

By substituting equation (14) and equation (4) into equation (10), the result is

$$
\begin{aligned}
& \Delta \mathrm{Z}= \\
& \int_{0}^{\mathrm{t}} \int_{0}^{\mathrm{R}_{\mathrm{C}}} \frac{2 \mathrm{kv \pi} \mathrm{Q}_{\mathrm{o}} \mathrm{R}_{1}}{\mathrm{R}_{\mathrm{c}}} \sqrt{\mathrm{R}_{\mathrm{c}}^{2}-\mathrm{R}_{1}^{2}} \sqrt{1+\frac{16 \mathrm{C}^{2} \mathrm{I}_{\max }^{2} \mathrm{R}_{1}^{2}}{\mathrm{~W}^{4}} \mathrm{e}^{-\frac{4 \mathrm{R}_{1}^{2}}{\mathrm{~W}^{2}}}} \mathrm{dR}_{1} \mathrm{dt} \\
& \because R_{l} \ll W
\end{aligned}
$$




$$
\therefore e^{-\left(\frac{R_{1}}{W}\right)^{2}} \cong 1
$$

Therefore, the equation (15) becomes

$\int_{0}^{t} \int_{0}^{R_{C}} \frac{2 k v \pi Q_{o} R_{1}}{R_{C}} \sqrt{R_{C}^{2}-R_{1}^{2}} \sqrt{1+\frac{16 C^{2} I_{\max }^{2} R_{1}^{2}}{W^{4}}} d R_{1} d t \quad(16)$
Considering that $R_{l} \ll W$, by Series Expansion of $\sqrt{1+\frac{16 \mathrm{C}^{2} \mathrm{I}_{\max }^{2} \mathrm{R}_{1}^{2}}{\mathrm{~W}^{4}}}$, and ignoring the higher powers and $\left(\frac{\mathrm{R}_{1}}{\mathrm{~W}}\right)^{4}$, the result is:

$$
\sqrt{1+\frac{16 C^{2} I_{\max }^{2} R_{1}^{2}}{W^{4}}} \cong 1+\frac{8 C^{2} I_{\max }^{2} R_{1}^{2}}{W^{4}}
$$

By substituting equation (17) into equation (16), the result is

$$
\begin{gathered}
\Delta Z=\int_{0}^{t} \int_{0}^{R_{C}^{2}} \frac{k v \pi Q_{o}}{R_{c}}\left(\sqrt{R_{c}^{2}-R_{1}^{2}}+\right. \\
\left.\frac{8 C^{2} I_{\max }^{2} R_{1}^{2} \sqrt{R_{C}^{2}-R_{1}^{2}}}{W^{4}}\right) d R_{1}^{2} d t
\end{gathered}
$$

After integration,

$$
\begin{aligned}
& \Delta \mathrm{Z}=\int_{0}^{t} \frac{k v \pi Q_{0}}{R_{c}}\left(\frac{2 R_{c}^{3}}{3}+\frac{32 C^{2} I_{\max }^{2} R_{c}^{5}}{15 W^{4}}\right) d t=\int_{0}^{t} \frac{2 k v \pi Q_{o} R_{c}^{2}}{3}(1+ \\
& \left.\frac{16 C^{2} I_{\max }^{2} R_{c}^{2}}{5 W^{4}}\right) d t
\end{aligned}
$$

According to elastic mechanics [3], the centre contact stress between the unit body and the brake pad is also the maximum contact stress $Q_{0}$ on the contact surface of pairs, which is:

$$
Q_{o}=\frac{3 P}{2 \pi R_{c}^{2}}
$$

By substituting equation (20) into equation (19), the result is

$$
\begin{aligned}
\Delta Z & =\int_{0}^{t} k v p\left(1+\frac{16 C^{2} I_{\max }^{2} R_{C}^{2}}{5 W^{4}}\right) d t \\
\Delta Z & =\int_{0}^{t} k v p(1+D) d t
\end{aligned}
$$

Where,

$$
D=\frac{16 C^{2} I_{\max }^{2} R_{C}^{2}}{5 W^{4}}
$$

Consequently, according to the equation (21), the Influence law of the laser-processed bionic brake drum unit body on the friction wear of the brake pad has been obtained.

\section{Conclusions}

By comparing the equation (22) and equation (5), it is indicated that the two equations are very similar. For the contact friction between two horizontal plane surfaces, the pressure $p_{n}$ in the equation (5) is along the vertical direction and is a constant. But for the pressure between two non-planar surfaces in contact, its value and direction are various with its position on the contact surface. It is a variable, which causes the integral in the equation (22) to add a term $D$ compared with the integral in the equation (5). Because $k, v$, and $P$ are all greater than 0 , thus if $D>0$, the $\Delta Z$ calculated by the equation (22) is greater than the one calculated by the equation (5). Which means the friction wear of the unit body of the bionic brake drum to the brake pad is greater than that of the non-bionic brake drum.

For normal non-bionic brake drum, its contact surface with the brake pad can be approximately judged as a plane contact, and the pressure is perpendicular to their contact surface. After implementation of the bionic brake drum in the brake, from a macro point of view, the pressure between the brake drum and the brake pad shows no change. However, due to the addition of small convex laser-processing bionic unit bodies on the bionic brake drum, the contact surface between the brake drum and the brake pad is no longer a plane contact. The value and direction of the pressure between the contact surfaces have become variables, and the original surface pressure cannot represent the real contact pressure. Since the contact surface is a Gaussian revolution surface, the contact surface is no longer perpendicular to the applied braking pressure, and the contact pressure between the surfaces will be increased, which increasing the friction between the contact surfaces as well as the wear of the brake pad. This is one of the main reasons why the bionic brake drum increases friction and wear on the brake pad.

Based on the equation (23), it is indicated that $D$ must be greater than zero. $D$ is increased with the increase of $C, I_{\max }$ and $R_{c}$, but decreased with the increase of $W$. This draw the conclusion:

1. When laser processing the bionic brake drum unit body, the larger the height ratio coefficient $C$ of the unit body caused by the laser processing is, the bigger the friction wear of the processed unit body on the brake pad is;

2. When laser processing the bionic brake drum unit body, the greater the light intensity $I_{\max }$ at the centre of the laser spot is, the bigger the friction wear of the unit body on the brake pad is.

3. The larger the radius $R_{C}$ of the contact surface between the unit body on the surface of the drum and the brake pad is, the bigger the friction wear of the processed unit body on the brake pad is.

4. When the laser intensity of the processing unit body is relatively concentrated, the beam radius $\mathrm{W}$ when the light intensity is $13.5 \%$ of the maximum light intensity will be smaller, which will increase the wear of the brake pad by the unit body.

With the value of $C$ increasing, the height of the unit body is increased, the pressure between the unit body and the brake pad is increased in braking process, the friction force between them is increased, the braking effect is increased, the wear rate of the brake pad caused by the unit body is increased, the wear of the brake pad is more serious. The wear of brake pad increases rapidly with the increase of $C$ value which is a square relationship.

When laser processing the bionic brake drum unit body, the light intensity $I_{\max }$ at the centre of the laser spot increases, which will cause two effects. Firstly, it increases the protrusion height of the unit body and enhances the impact of the unit body in the braking process which increases the friction and wear caused by the unit body to the brake pad. Secondly, the laser is concentrated on the axis direction, so that the radius of the convex unit tip can be reduced which makes the unit body sharp. It is easier to penetrate the brake pad surface. With the depth of the unit body pressed into the surface of the brake pad increasing, the wear of the brake pad 
will be increased inevitably. The wear of brake pads increases faster with the increase of $I_{\max }$, which is a square relationship.

The larger the radius $R_{C}$ of the contact surface between the unit body on the surface of the brake drum and the brake pad is, the deeper the unit body is pressed into the brake pad, the bigger pressure between them is, the more serious their friction is, the more quickly the wear of brake pad is increased. The wear of brake pad is increased faster than the increase of $R_{C}$ value, which is a square relationship.

With the light beam radius $W$ when the light intensity is $13.5 \%$ of the maximum light intensity decreasing, it means that the laser beam intensity for processing the bionic brake drum unit body is relatively concentrated, the top of the unit body is sharper, and the top arc radius of the unit body is reduced. It is easy to pierce the surface of the brake pad, and the unit body presses the surface of the brake pad more deeply, which will increase the wear of the brake pad. The wear of brake pad increases rapidly with the increase of $W$ value, showing a $4^{\text {th }}$ power relationship.

The interaction between the unit body on the bionic brake drum and the brake pad has been analysed; the contact stress distribution law of their interaction has been obtained, as well as the friction wear of the unit body to the brake pad according to the stress distribution law has been calculated. Regardless of other factors, the friction wear of the unit body on the brake pad will be increased considering the change of surface feature only. Although some assumptions and approximations have been made which leads the certain errors, the trend of change is confirmed. To sum up, the unit body will definitely increase the friction wear of the brake pad.

In order to improve the environmental friendliness of the brake drum and reduce the wear of the brake pad, the following aspects should be considered. Firstly, the height of the bionic brake drum unit body should be reduced during laser processing; Secondly, when laser processing the bionic brake drum unit body, the light intensity at the centre of the laser spot should be decreased; At last, the adjustment of the laser beam intensity distribution is necessary to make the intensity distribution more uniform during laser processing.

\section{References}

1. J Dong. Influence of laser alloy bionic unit with $\mathrm{WC} / \mathrm{Cr}$ on fatigue wear resistance of gray cast iron. Jilin University, Changchun (2016)

2. $\mathrm{Z}$ Liang. The effects of alloying enhanced bionic units on the tensile properties of low-carbon steel, Jilin University, Changchun (2013)

3. T Li; S Yin. Tan Su Xing Li Xue. China University of Geosciences. Wuhan. 212-215 (1990).

4. S Ji. Study on Machinability of Softness Abrasive Flow Based on Preston Equation. JOURNAL OF MECHANICAL ENGINEERING, 17.156-163(2011). 